\title{
A new lower bound approach for single- machine multicriteria scheduling
}

\author{
J.A. Hoogeveen \\ Centre for Mathematics and Computer Science, P.O. Box 4079, 1009 AB Amsterdam, The Netherlands \\ S.L. van de Velde \\ School of Management Studies, Unicersity of Twente, P.O. Box 217, 7500 AE Enschede, The Netherlands
}

Received September 1990

Revised February 1991

The concept of maximum potential improvement has played an important role in computing lower bounds for single-machine scheduling problems with composite objective functions that are linear in the job completion times. We introduce a new method for lower bound computation: objective splitting. We show that it dominates the maximum potential improvement method in terms of speed and quality.

single-machine scheduling; multicriteria scheduling; maximum potential improvement; objective splitting

\section{Introduction}

A single-machine job shop can be described as follows. A set of $n$ independent jobs has to be scheduled on a single machine that is continuously available from time zero onwards and that can process no more than one job at a time. Each job $J_{i}(i=1, \ldots, n)$ requires processing during a positive time $p_{i}$. In addition, it has a due data $d_{i}$, at which it should ideally be completed. A schedule defines for each job $J_{i}$ its completion time $C_{i}$ such that no two jobs overlap in their execution. A performance measure or scheduling criterion associates a value $f(\sigma)$ with each feasible schedule $\sigma$. Some well-known measures are the sum of the job completion times $\Sigma C_{i}$, the maximum job lateness $L_{\max }=\max _{1 \leqslant i \leqslant n}\left(C_{i}-d_{i}\right)$, and the maximum job earliness $E_{\max }=\max _{1 \leqslant i \leqslant n}\left(d_{i}-C_{i}\right)$.

In this paper, we adopt the terminology of Graham, Lawler, Lenstra and Rinnooy Kan (1979) to classify scheduling problems. Scheduling problems are classified according to a three-field notation $\alpha|\beta| \gamma$, where $\alpha$ specifies the machine environment, $\beta$ the job characteristics, and $\gamma$ the objective function. For instance, $1|\mathrm{nmit}| E_{\max }$ denotes the single-machine problem of minimizing maximum earliness, where nmit denotes that no machine idle time is allowed.

Most research has been concerned with a single criterion. In real life scheduling, however, it is necessary to take several performance measures into account. There are basically two approaches to cope with multiple criteria. If the scheduling criteria are subject to a welldefined hierarchy, they can be considered sequentially in order to relevance. An example is the problem of minimizing maximum lateness subject to the minimum number of tardy jobs, for which Shanthikumar (1983) presents a branch-and-bound algorithm.

The second approach is simultaneous optimization of several criteria. The $K$ performance measures specified by the functions $f_{k}(k=$ $1, \ldots, K)$ are then transformed into one single composite objective function $F: \Omega \rightarrow \mathbb{R}$, where $\Omega$ denotes the set of all feasible schedules. We restrict ourselves to the case that $F$ is a linear 
composition of the individual performance measures. This leads to the problem class $(P)$ that contains all problems that can be formulated as

$\min _{\sigma \in \Omega} \sum_{k=1}^{K} \alpha_{k} f_{k}(\sigma)$

where $\alpha=\left(\alpha_{1}, \ldots, \alpha_{K}\right)$ is a given vector of real nonnegative weights. The problem of minimizing a linear function of the number of tardy jobs and maximum lateness, denoted as $1 \| \sum U_{i}+L_{\max }$, is a member of this class. Nelson, Sarin and Daniels (1986) present a branch-and-bound algorithm for its solution.

In addition to solving some problem in $(\mathrm{P})$ for a given $\alpha \geqslant 0$, it may be of interest to determine the extreme set. The extreme set for given functions $f_{1}, \ldots, f_{K}$ is defined as the minimum cardinality set that contains an optimal schedule for any weight vector $\alpha \geqslant 0$. The elements of this set are the extreme schedules. If this set has been identified, then we can solve any problem for these functions by computing the function value for each extreme schedule and choosing the best. Hence, if the cardinality of the extreme set is polynomially bounded in $n$, the number of jobs, and if each extreme schedule can be found in polynomial time, then any problem in $(P)$ with respect to these functions $f_{1}, \ldots, f_{K}$ can be solved in polynomial time.

Suppose that some problem in (P) is NP-hard and that one wishes to design a branch-and-bound method for its solution. In that case, good lower bounds are required. Unil now, virtuallly all lower bound computations for problems in $(\mathrm{P})$ are based upon the so-called maximum potential improvement method. We prove in Section 2 that these bounds are dominated in terms of quality and computational effort by a much simpler method that we name objective splitting. In Section 3 , we refine the basis objective splitting method.

The problem 1||$\sum C_{i}+L_{\max }+E_{\max }$ is our benchmark in comparing the two lower bound approaches. It is still an open question whether this problem is NP-hard. Sen, Raiszadeh and Dileepan (1988) develop a branch-and-bound algorithm and derive lower bounds by means of the maximum potential improvement method. There is an optimal schedule for this problem without machine idle time, although $E_{\max }$ is nonincreasing in the job completion times. It is not mean- ingful to insert idle time, as the gain for $E_{\max }$ will at least be compensated by the increase of $\Sigma C_{i}$. We recall the following fundamental algorithms for the three embedded subproblems.

Theorem 1 (Smith, 1956). The 1||$\Sigma C_{i}$ problem is minimized by sequencing the jobs according to the shortest-processing-time (SPT) rule, that is, in order of nondecreasing $p_{i}$.

Theorem 2 (Jackson, 1955). The 1||$L_{\max }$ problem is minimized by sequencing the jobs according to the earliest-due-date $(E D D)$ rule, that is, in order of nondecreasing $d_{i}$.

Theorem 3. The $1|\mathrm{nmit}| E_{\max }$ problem is solved by sequencing the jobs according to the minimumslack-time (MST) rule, that is, in order of nondecreasing $d_{i}-p_{i}$.

The proof of each of these algorithms proceeds by a straightforward interchange argument. Note that each of these problems is solved by arranging the jobs in a certain priority order that can be specified in terms of the parameters of the problem type.

The optimal solution values for these singlemachine scheduling problems will be denoted by $\sum C_{i}^{*}, L_{\max }^{*}$ and $E_{\max }^{*}$, respectively. Furthermore, $\Sigma C_{i}(\sigma), L_{\max }(\sigma)$, and $E_{\max }(\sigma)$ are the objective values for the schedule $\sigma$. In analogy, $C_{i}(\sigma)$, $L_{i}(\sigma)$, and $E_{i}(\sigma)$ denote the respective measures for job $J_{i}(i=1, \ldots, n)$. Whenever $(\sigma)$ is omitted, we are considering the performance measure in a generic sense, or there is no confusion possible as to the schedule we are referring to. The schedules that minimize $\Sigma C_{i}, L_{\max }$, and $E_{\max }$ are referred to as SPT, EDD, and MST respectively. In addition, $\nu(\cdot)$ denotes the optimal objective value for problem $\cdot$.

\section{Maximum potential improvement versus ob- jective splitting}

Townsend (1978) proposed the maximum potential improvement method to compute lower bounds for minimizing a quadratic function of the job completion times. Since then, the method has been extended to problems in (P), including $1 \mid \Sigma C_{i}+L_{\max }$ (Sen and Gupta, 1983), 1 |nmit $\mid$ 
$L_{\max }+E_{\max }$ (Gupta and Sen, 1984), and 1||$\Sigma C_{i}$ $+L_{\max }+E_{\max }$ (Sen, Raiszadeh, and Dileepan, 1988). To our knowledge, there is only one publication on objective splitting avant la lettre: Tegze and Vlach (1988) obtained an extremely simple, but provably stronger lower bound for $1 \mid$ nmit $\mid L_{\max }+E_{\max }$.

Meanwhile, Hoogeveen (1990) and Hoogeveen and Van de Velde (1990) have found polynomial-time algorithms for $1|\mathrm{nmit}| \alpha_{1} L_{\max }+\alpha_{2} E_{\max }$ and 1||$\alpha_{1} \sum C_{\mathrm{i}} \mathrm{i}+\alpha_{2} L_{\max }$. The former problem has $\mathrm{O}(n)$ extreme schedules, each of which is found in $\mathrm{O}(n \log n)$ time. The latter problem has $\mathrm{O}\left(n^{2}\right)$ extreme schedules, each of which is determined in $\mathrm{O}(n)$ time after appropriate preprocessing. However, it is an interesting issue how to derive lower bounds for NP-hard problems in (P). The maximum potential improvement method is a cumberstone procedure. However, by viewing it from a different angle, we derive a closed expression for the resulting lower bound. It is then immediately clear that the maximum potential improvement method is completely dominated by the much simpler objective splitting method.

Objectice splitting is based upon the observation that

$$
\begin{gathered}
\min _{\sigma \in \Omega}\left[\sum_{k=1}^{K} \alpha_{k} f_{k}(\sigma)\right] \\
\geqslant \sum_{k=1}^{K} \alpha_{k}\left[\min _{\sigma \in \Omega} f_{k}(\sigma)\right],
\end{gathered}
$$

if $\alpha_{k} \geqslant 0$ for $k=1, \ldots, K$. The application of this idea to 1||$\Sigma C_{i}+L_{\max }+E_{\max }$ yields the problems 1||$\sum C_{i}, 1|| L_{\max }$, and $1|\mathrm{nmit}| E_{\max }$. Each problem is polynomially solvable, and we obtain the $L B^{\mathrm{OS}}=\Sigma C_{i}^{*}+L_{\max }^{*}+E_{\max }^{*}$. This bound is computed in $\mathrm{O}(n)$ time in each node of the search tree, provided that the SPT, EDD, and MST sequences have been stored and that we employ a convenient branching strategy.

It is relatively easy to apply the maximum potential improvement method to problems in ( $\mathrm{P})$ for which each embedded single-machine problem has a priority order. The 1||$\sum C_{i}+L_{\max }+$ $E_{\max }$ problem has three: the SPT order for $\Sigma C_{i}$, the EDD order for $L_{\max }$, and the MST order for $E_{\max }$. Clearly, we have solved an instance of this problem in case these orders concur; in general though, the priority orders are conflicting.
Suppose we start with the MST schedule, which we refer to as the primary priority order. The scheduling cost induced by the MST schedule is $\Sigma C_{i}(\mathrm{MST})+E_{\max }^{*}+L_{\text {max }}(\mathrm{MST})$; this is obviously an upper bound on the optimal solution value. In addition, we know that any optimal schedule $\sigma^{*}$ must have $E_{\max }\left(\sigma^{*}\right) \geqslant E_{\max }^{*}$, and $\Sigma C_{i}\left(\sigma^{*}\right)+$ $L_{\max }\left(\sigma^{*}\right) \leqslant \Sigma C_{i}(\mathrm{MST})+L_{\max }(\mathrm{MST})$. The maximum potential improvement method assesses the current schedule with respect to the maximum improvement that can be obtained for each of the performance measure separately. Accordingly, we get a lower bound by subtracting the total maximum potential improvement from the upper bound.

First, consider the maximum lateness criterion, which is the secondary priority order. If we interchange every pair of adjacent jobs $J_{i}$ and $J_{j}$ for which $d_{i}>d_{j}$ and $C_{i}<C_{j}$, then we need to conduct $\mathrm{O}\left(n^{2}\right)$ interchanges before we have transformed the MST schedule into an EDD schedule. The actual effect on the objective value by one particular interchange depends on the interchanges that have been conducted thusfar. It might have no effect whatsoever on the performance of the schedule; this is true if both the maximum lateness and the maximum earliness remain unchanged. The maximum possible decrease of the scheduling cost, however, is $d_{i}-d_{j}$; if $\sigma$ and $\pi$ denote the schedule before and after the interchange, respectively, then the maximum decrease is realized if $L_{\max }(\sigma)=L_{j}(\sigma), L_{\max }(\pi)$ $=L_{i}(\pi)$ and $E_{\max }(\pi)=E_{\mathrm{max}}(\sigma)$. The effect that the interchange might have on the sum of the job completion times is not considered here and dealt with separately. Any interchange conducted to transform the MST schedule into the EDD schedule may improve the maximum lateness by the corresponding maximum possible decrease. The sum of these is the maximum potential improvement with respect to the initial lateness $L_{\text {max }}(\mathrm{MST})$. It is given by

$$
\mathrm{MPI}_{2}=\sum_{i, j: d_{i}>d_{j}, C_{i}<C_{j}}\left(d_{i}-d_{j}\right) \text {. }
$$

Note that the maximum potential improvement does not depend on the order in which the interchanges are conducted.

Second, the sum of the job completion times, which is the tertiary priority order, is reduced by interchanging two adjacent jobs $J_{i}$ and $J_{j}$ with 
$p_{i}>p_{j}$ and $C_{i}<C_{j}$. The maximum potential improvement is then $p_{i}-p_{j}$, which is also the true improvement. The maximum potential improvement with respect to $\Sigma C_{j}(\mathrm{MST})$ is then

$\mathrm{MPI}_{3}=\sum_{i, j: p_{i}>p_{j}, C_{i}<C_{j}}\left(p_{i}-p_{j}\right)$.

The lower bound LB $^{\text {MPI }}$ suggested by Sen, Raiszadeh and Dileepan (1988) for 1||$\Sigma C_{i}+$ $L_{\text {max }}+E_{\max }$ is then

$$
\begin{aligned}
\mathrm{LB}^{\mathrm{MPI}}= & E_{\max }^{*}+L_{\max }(\mathrm{MST})-\mathrm{MPI}_{2} \\
& +\Sigma C_{i}(\mathrm{MST})-\mathrm{MPI}_{3} .
\end{aligned}
$$

Since $\Sigma C_{i}(\mathrm{MST})-\mathrm{MPI}_{3}=\Sigma C_{i}(\mathrm{SPT})=\Sigma C_{i}^{*}$ and $L_{\max }(\mathrm{MST})-\mathrm{MPI}_{3} \leqslant L_{\text {max }}^{*}$, as we have systematically overestimated the reduction in maximum lateness, we conclude that

$$
\begin{aligned}
\mathrm{LB}^{\mathrm{MPI}} & =E_{\max }^{*}+\Sigma C_{i}^{*}+L_{\max }(\mathrm{MST})-\mathrm{MPI}_{2} \\
& \leqslant \mathrm{LB}^{\mathrm{OS}} .
\end{aligned}
$$

The maximum potential improvement method can be generalized to problems in (P) as follows. Let $\sigma_{k}^{*}$ denote an optimal schedule for the $k$-th individual objective. Furthermore, let the optimal sequence that goes with the $k$-th objective be the $k$-th preference order. The first step is then to sequence the jobs according to the primary preference order, which gives the upper bound $\alpha_{1} f_{1}\left(\sigma_{1}^{*}\right)+\sum_{k=2}^{K} \alpha_{k} f_{k}\left(\sigma_{1}^{*}\right)$. We then have to transform the primary preference order into the $k$-th preference order for $k=2, \ldots, K$, and determine the corresponding maximum potential improvement MPI $k$. The lower bound is then given by

$$
\mathrm{LB}^{\mathrm{MPI}}=\alpha_{1} f_{1}\left(\sigma_{1}^{*}\right)+\sum_{k=2}^{K} \alpha_{k}\left(f_{k}\left(\sigma_{1}^{*}\right)-\mathrm{MPI}_{k}\right) .
$$

Note that this procedure requires $\mathrm{O}\left(n^{2}\right)$ time for fixed $K$ in addition to the time required to determine $\sigma_{k}^{*}$, for $k=1, \ldots, K$. Since $f_{k}\left(\sigma_{1}^{*}\right)-\mathrm{MPI}_{k}$ $\leqslant f_{k}\left(\sigma_{k}^{*}\right)$ for each $k=1, \ldots, K$, we have the following theorem.

Theorem 4. For any problem in $(\mathrm{P})$, the lower bound obtained by the maximum potential improvement method is dominated in terms of both quality and speed by the lower bound obtained by the objective splitting method.

Consider the following example shown in Table 1 that is taken from Sen, Raiszadeh and Dileepan
Table 1

\begin{tabular}{lrrrr}
\hline & $J_{i}$ & & & \\
\cline { 2 - 5 } & $J_{1}$ & $J_{2}$ & $J_{2}$ & \multicolumn{1}{c}{$J_{4}$} \\
\hline$P_{i}$ & 14 & 7 & 6 & 7 \\
$d_{i}$ & 20 & 14 & 15 & 17 \\
$d_{i}-p_{i}$ & 6 & 7 & 9 & 10 \\
\hline
\end{tabular}

(1988) for the problem 1||$q \sum C_{i}+(1-q)\left(L_{\max }\right.$ $+E_{\max }$ ) with $0 \leqslant q \leqslant 1$. By means of the maximum potential improvement method, we obtain the lower bound $\mathrm{LB}^{\mathrm{MPI}}=64 q+9$. It is easy to verify that $\sum C_{j}^{*}=73, L_{\max }^{*}=14$, and $E_{\max }^{*}=6$. This gives the bound $\mathrm{LB}^{\mathrm{OS}}=53 q+20$. Note that $53 q+20 \geqslant 64 q+9$ for all $q$ with $0 \leqslant q \leqslant 1$.

\section{Improving the objective splitting procedure}

The objective splitting procedure above was given in its simplest form: we separated the composite objective function into $K$ single-criterion scheduling problems. We now propose a refinement that gives us a lower bound that is at least as good, but requires more time. Our more general approach allows combinations of objective functions. Let $\left(T_{1}, \ldots, T_{H}\right)$ be a partition of the set $\{1, \ldots, K\}$, i.e., the sets $T_{h}$ are mutually disjoint and $\cup_{h=1}^{H} T_{h}=\{1, \ldots, K\}$. For any problem $A$ in the class $(P)$ we clearly have

$$
\begin{aligned}
\nu(A) & \geqslant \sum_{h=1}^{H}\left[\min _{\sigma \in \Omega} \sum_{k \in T_{h}} \alpha_{k} f_{k}\left(\sigma_{k}\right)\right] \\
& \geqslant \sum_{k=1}^{K} \alpha_{k}\left[f_{k}\left(\sigma_{k}^{*}\right)\right]=\mathrm{LB}^{\mathrm{OS}} .
\end{aligned}
$$

This idea can be refined even further, since it is not obligatory to match each performance criterion $f_{k}$ with only one set $T_{h}$. Hence, let us relax the assumption that $\left(T_{1}, \ldots, T_{H}\right)$ is a partition of $\{1, \ldots, K\}$, and let $\alpha_{k h}$ denote the fraction of $f_{k}$ that is assigned to $T_{h}$. We must have that $\sum_{h} \alpha_{k h}$ $=\alpha_{k}$ for each $k=1, \ldots, K$, and also that $\alpha_{k h} \geqslant 0$, since the composite objective function associated with the set $S_{h}$ has to be nondecreasing in each of its arguments, for $h=1, \ldots$, . We can compute the lower bound for given values of $\alpha_{k h}$ as

$$
\nu(\mathrm{OS})=\sum_{h=1}^{H}\left[\min _{\sigma \in \Omega} \sum_{k \in T_{h}} \alpha_{k h} f_{k}(\sigma)\right] .
$$


An interesting question is how to determine the values of $\alpha_{k h}$ that maximize the lower bound $\nu(\mathrm{OS})$. This problem, referred to as problem (D), is to

(D) maximize $\nu$ (OS)

subject to

$$
\begin{aligned}
& \sum_{h=1}^{H} \alpha_{k h}=\alpha_{k} \quad \text { for } k=1, \ldots, K, \\
& \alpha_{k h} \geqslant 0 \quad \text { for } k=1, \ldots, K, h=1, \ldots, H .
\end{aligned}
$$

A sufficient condition for solving problem (D) in polynomial time (for fixed $K$ ) is that the extreme set for each problem induced by $T_{h}(h=1, \ldots, H)$ can be determined in polynomial time. In that case, there is only a polynomial number of extreme schedules involved, and problem (D) can then be formulated as a linear programming problem with a polynomial number of constraints and variables. Let $N(h)$ be the number of extreme schedules for the problem associated with $T_{h}(h=1, \ldots, H)$, and let $\sigma_{j(h)}$ denote the $j$-th extreme schedule for the problem associated with $T_{h}$. There are at most $2^{K}-2$ sets $T_{h}\left(\left|T_{h}\right|<K\right.$ and $T_{h} \neq \emptyset$ ). The linear program is then to

\section{maximize $w$}

subject to

$$
\begin{aligned}
& w \leqslant \sum_{h=1}^{H} \sum_{k \in T_{h}} \alpha_{k h} f_{k}\left(\sigma_{j(j)}\right) \\
& \quad \text { for } j(h)=1, \ldots, N(h), h=1, \ldots, H, \\
& \sum_{h=1}^{H} \alpha_{k h}=\alpha_{k} \text { for } k=1, \ldots, K, \\
& \alpha_{k h} \geqslant 0 \text { for } k=1, \ldots, K, h=1, \ldots, H .
\end{aligned}
$$

In general, it would be unreasonable to presume that each of the possible $2^{K}-2$ sets $T_{h}$ would result into a polynomially solvable problem; it may be a formidable challenge to identify those that will. If we touch upon a problem that appears to be hard to solve, then we may relax the assumptions by allowing preemption (I.e., the processing of the jobs may be interrupted and resumed to the computational complexity, but also with respect to the lower bound quality. The latter follows particularly from the following theorem.
Theorem 6. The optimal objective value of $1|\mathrm{pmtn}| \sum_{k=1}^{K} \alpha_{k} f_{k}$ is greater than or equal to $\sum \alpha_{k} f_{k}\left(\sigma_{k}^{*}\right)$, where $\sigma_{k}^{*}$ is the optimal value for 1||$f_{k}(k=1, \ldots, K)$.

Proof. The proof follows from the observation that $\sigma_{k}^{*}$ also solves $1|\mathrm{pmtn}| f_{k}$, if $f_{k}$ is either monotonically nondecreasing or monotonically nonincreasing in the job completion times.

If we apply the refined objective splitting procedure to 1||$\Sigma C_{i}+L_{\max }+E_{\max }$, then, except for the obvious single-criterion problems, we have to consider three problems: 1||$\alpha_{1} \Sigma C_{i}+\alpha_{2} L_{\max }$, $1|\mathrm{nmit}| \alpha_{1} \Sigma C_{i}+\alpha_{2} E_{\max }$, and $1|\mathrm{nmit}| \alpha_{1} L_{\max }+$ $\alpha_{2} E_{\max }$. Hoogeveen (1990) presents and $\mathrm{O}\left(n^{2} \log n\right)$ time algorithm for $1|\mathrm{nmit}| \alpha_{1} L_{\max }+$ $\alpha_{2} E_{\max }$ to find the $\mathrm{O}(n)$ extreme schedules, and Hoogeveen and Van de Velde (1990) present and $\mathrm{O}\left(n^{3}\right)$ time algorithm for 1||$\alpha_{1} \Sigma C_{i}+\alpha_{2} L_{\max }$, which has $\mathrm{O}\left(n^{2}\right)$ extreme schedules. For the problem (Hoogeveen and Van de Velde, 1990). The complexity of the case $\alpha_{1}<\alpha_{2}$ is unknown. However, $1 \mid$ nmit, pmtn $\mid \alpha_{1} \sum C_{i}+\alpha_{2} E_{\max }$ is solvable in $\mathrm{O}\left(n^{4}\right)$ time and has $\mathrm{O}\left(n^{2}\right)$ extreme schedules.

If we reconsider the example, we find that there is one extreme schedule for $\sum C_{i}$ and $L_{\max }$ with $\Sigma C_{i}=73$ and $L_{\max }=14$; there are two extreme schedules for $L_{\max }$ and $E_{\max }$ with values $L_{\max }=14$ and $E_{\max }=7$, and $L_{\max }=17$ and $E_{\max }$ $=6$; there are three extreme schedules for $E_{\max }$ and $\Sigma C_{i}$ if we allow preemption with values $E_{\max }$ $=6$ and $\Sigma C_{i}=96, E_{\max }=7$ and $\Sigma C_{i}=74$, and $E_{\max }=9$ and $\Sigma C_{i}=73$, respectively.

The lower bound that is obtained by the improved objective splitting method depends on the parameter $q$. Suppose $q=\frac{1}{2}$. Then we obtain $\mathrm{LB}^{\mathrm{MPI}}=41$ and $\mathrm{LB}^{\mathrm{OS}}=46 \frac{1}{2}$. It is easy to verify that the improved objective splitting method gives $47 \frac{1}{2}$ as a lower bound. This bound is tight, since the optimal sequence $\left(J_{2}, J_{3}, J_{4}, J_{1}\right)$ has the same value.

\section{Acknowledgement}

The authors are grateful to Jan Karel Lenstra for his comments on earlier drafts of this paper. 


\section{References}

R.L. Graham, E.L. Lawler, J.K. Lenstra and A.H.G. Rinnooy Kan (1979), "Optimization and approximation in deterministic sequencing and scheduling: A survey", Ann. Discrete Math. 5, 287-326.

S.K. Gupta and T. Sen (1984), "Minimizing the range of lateness on a single machine", J. Oper. Res. Soc. 35, 853-857.

J.A. Hoogeveen (1990), "Minimizing maximum earliness and maximum lateness on a single machine," Report BSR9001, Centre for Mathematics and Computer Science, Amsterdam.

J.A. Hoogeveen and S.L. van de Velde (1990), "Polynomialtime algorithms for multicriteria scheduling problems", Report BS-R9008, Centre for Mathematics and Computer Science, Amsterdam.

J.R. Jackson (1955), "Scheduling a production line to minimize maximum tardiness", Research Report 43, Management Sciences Research Project, UCLA, Los Angeles, CA.
R.T. Nelson, R.K. Sarin and R.L. Daniels (1986), "Scheduling with multiple performance measures: The one-machine case", Management Sci. 32, 464-479.

T. Sen and S.K. Gupta (1983), A branch-and-bound procedure to solve a bricriterion scheduling problem", IIE Trans. 15, 84-88.

T. Sen, F.M.E. Raiszadeh and P. Dileepan (1988), "A branchand-bound approach to the bicriterion scheduling problem involving total flowtime and range of lateness", Management Sci. 34, 254-260.

J.G. Shanthikumar (1983), "Scheduling $n$ jobs on one machine to minimize the maximum tardiness with minimum number tardy", Comput. Oper. Res. 10, 255-266.

W.E. Smith (1956), "Various optimizers for single-stage production. Naval Res. Logist. Quart. 1, 59-66.

M. Tegze and M. Vlach (1988), "Improved bounds for the range of lateness on a single machine", J. Oper. Res. Soc. 39, 675-680.

W. Townsend (1978), "The single machine problem with quadratic penalty function of completion times: a branchand-bound solution", Management Sci. 24, 530-534. 